Décadrages Décadrages

cinéma, à travers champs Cinéma, à travers champs

$28 \mid 2014$

Arnaud Desplechin

\title{
Le clivage du sujet, ou les exercices de style d'Arnaud Desplechin
}

\section{François Bovier et Cédric Fluckiger}

\section{(2) OpenEdition}

1 Journals

\section{Édition électronique}

URL : http://journals.openedition.org/decadrages/760

DOI : $10.4000 /$ decadrages. 760

ISSN : 2297-5977

Éditeur

Association Décadrages

\section{Édition imprimée}

Date de publication : 10 octobre 2014

Pagination : 8-21

ISBN : 978-2-9700668-9-7

ISSN : 2235-7823

Référence électronique

François Bovier et Cédric Fluckiger, «Le clivage du sujet, ou les exercices de style d'Arnaud Desplechin ", Décadrages [En ligne], 28 | 2014, mis en ligne le 11 janvier 2016, consulté le 09 octobre 2020. URL : http://journals.openedition.org/decadrages/760 ; DOI : https://doi.org/10.4000/decadrages.760 


\section{Le clivage du sujet, ou les exercices de style d'Arnaud Desplechin}

1 Voir notamment Camille Nevers, «La Relève», Cahiers du cinéma, n 457 , juin I992, pp. I2-I4; Antoine de Baecque, «Le Livre ouvert», Cahiers du cinéma, $\mathrm{n}^{\circ}$ 503, juin i996, pp. 26-29; Emmanuel Burdeau, «Hold-up romanesque», $\mathrm{Ca}$ hiers du cinéma, hors-série $\mathrm{n}^{\circ} 22$ ( «Nouvelle Vague: une légende en question»), décembre i998, pp. 67-69. Voir aussi Marja Warehime, "Politics, Sex and French Cinema in the iggos: the Place of Arnaud Desplechin", French Studies, vol. $56, \mathrm{n}^{\circ} \mathrm{I}$, janvier 2002, pp. 6I-78.

2 Notons que Versailles Rive-Gauche (France, I992) de Bruno Podalydès présente d'évidentes affinités avec l'univers satirique et caustique de Desplechin.

3 Pour une charge virulente contre le formalisme «moderniste» et la cinéphilie «masculine» des critiques qui prennent la défense du «cinéma d'auteur», lire Noël Burch, De la beauté des latrines. Pour réhabiliter le sens au cinéma et ailleurs, Paris, L'Harmattan, 2007. Rappelons que la «fonction-auteur» est théorisée par Foucault dès I969 ("Qu'est-ce qu'un auteur?», Dits et écrits, tome I, Paris, Gallimard, I994, pp. 789-82I) - tandis que Barthes annonce sa mort en i968 («La mort de l'auteur», Le Bruissement de la langue: Essais critiques IV, Paris, Seuil, I984, pp. 63-69).
Les Films D’Arnaud Desplechin s'apparentent à une série d'exercices de style virtuoses, qui s'approprient différents genres codifiés: le film d'espionnage (La Sentinelle, France, I992), la comédie de mours (Comment je me suis disputé... (ma vie sexuelle), France, I996), la comédie dramatique (Rois et Reine, France, 2004), ou encore le biopic (Jimmy P., France/Etats-Unis, 2013). Ils reposent sur un travail en équipe: Desplechin a su réunir autour de ses productions une troupe d'acteurs, dont certains sont présents dès son premier moyen métrage, La Vie des morts (France, I99I, en l'occurrence Marianne Denicourt, Emmanuelle Devos, Emmanuel Salinger, Thibault de Montalembert); et il collabore régulièrement avec les mêmes scénaristes ou adaptateurs (Emmanuel Bourdieu, Noémie Lvovsky, Pascale Ferran, Emmanuel Seignier), ainsi qu'avec un chef-opérateur régulier (Eric Gautier). Rapidement investi comme le principal acteur du renouvellement de l'après-Nouvelle Vague, Desplechin incarne le «cinéma d'auteur» par excellence, du moins aux yeux de la critique ${ }^{1}$. Précisons toutefois que contrairement à cette optique cinéphilique française, les premiers longs métrages de Desplechin, sur lesquels nous nous arrêterons ici, se situent en rupture par rapport à l'énonciation distanciée et maniériste de films de la génération qui le précède immédiatement et dont l'a mbition affichée peut être caractérisée comme celle d'un nouvel objectivisme factice, à l'instar de Mauvais sang (France, 1986) de Leos Carax; dans une perspective diamétralement opposée, Desplechin s'approprie les conventions et le «style» du cinéma d'art et d'essai en vue d'effets comiques, une autodérision généralisée parasitant la logique de la citation révérencieuse et les stratégies du pastiche ${ }^{2}$. On vient de le signaler, les films de Desplechin se distinguent par un dialogue avec le cinéma de genre tout comme par une étonnante diversité dans leurs modalités d'énonciation, contrecarrant la constitution d'un style unifié, voire le déploiement d'une thématique récurrente, pourtant garants de l'«effet-auteur» ${ }^{3}$. La 
singularité du système ou du style Desplechin (sacrifions donc à notre tour à la fonction-auteur) repose sur cette dynamique de variations autour de canevas génériques prédéterminés, qui se signalent par une multiplicité de références culturelles et par le recours à la citation comme masque révélateur. Desplechin suggère lui-même une piste d'interprétation - mais ce faisant, livre-t-il une clef de lecture de ses films ou s’ingénie-t-il à brouiller les pistes? - en mobilisant la réflexion de Stanley Cavell sur le cinéma de genre hollywoodien, notamment dans le cadre d'émissions radio récentes ${ }^{4}$. Sur quel plan ce modèle opère-t-il donc?

\section{L'appropriation des conventions de genre du cinéma hollywoodien}

A l'occasion d'une rencontre organisée par Citéphilo à Lille en 2006, Arnaud Desplechin ne manque pas de placer explicitement ses films sous le signe du cinéma de genre tel que Cavell le redéfinit:

«Comme beaucoup de lecteurs français, j’ai d’abord découvert Stanley Cavell par son livre sur les comédies du remariage ${ }^{5}$. Cet ouvrage a été un outil de travail décisif pour moi pendant le tournage de Comment je me suis disputé... ${ }^{6}$

Il est possible de déceler dans les longs métrages de Desplechin l'influence des spéculations de Cavell sur le cinéma de genre, comme Elise Domenach l'expose à partir d'Un conte de Noël (France, 2008) vis-à-vis du «perfectionnisme» moral (dans la tradition de Ralph Waldo Emerson) ${ }^{7}$. Et le cinéaste l'affirme lui-même: Comment je me suis disputé... constitue une variation sur la «comédie de remariage», Esther Khan sur le «mélodrame de la femme inconnue ${ }^{8}$. De toute évidence, Desplechin joue sur les horizons d'attente liés à des conventions de genre, qu'il s'approprie en les confrontant à d'autres modalités de construction discursive et logiques de sens. Ses films enchâssent les récits à travers une énonciation qui privilégie le recours à la voix over et au flashback, exprimant

4 Voir les émissions de France-culture «La suite dans les idées» du 24 mai 2014 et «Les nouveaux chemins de la connaissance» du I3 septembre 2013 .

5 Voir Stanley Cavell, A la recherche du bonheur. Hollywood et la comédie du remariage, Paris, Editions de l'Etoile/Cahiers du cinéma, I993, trad. C. Fournier et S. Laugier [première édition: Pursuits of Happiness: The Hollywood Comedy of Remarriage, Cambridge, Ma./Londres, Harvard University Press, I98I]. Spécialiste du scepticisme et du transcendantalisme, partisan de la logique analytique et de la philosophie du langage, Cavell mobilise dans ses nombreux ouvrages de philosophie les outils de l'empirisme anglo-saxon dans une perspective élargie et renouvelée.

6 «Pourquoi les films comptent-ils? Discussion entre Stanley Cavell et Arnaud Desplechin», Esprit, nº 8-9, aoûtseptembre 2008, pp. 208-209, trad. Elise Domenach et Christian Fournier. Desplechin souligne la parenté de construction scénaristique entre Comment je me suis disputé... et la structure des comédies de remariage: «Je me souviens avoir demandé à Stanley Cavell, le jour où il dédicaçait Conditions nobles et ignobles à Paris: ‘Comment fait-on des comédies du remariage quand il n'y a pas de remariage possible?> Il m’a répondu en substance: «Même pour un catholique, il doit y avoir des moyens de divorcer et se remarier.) Cela m'avait été d'une grande aide.» (id., p. 2I2).

7 Voir Elise Domenach, «Un conte de Noël. La nouvelle Arcadie d'Arnaud Desplechin», id., pp. I9I-207. Domenach conçoit l'écriture filmique par notes, varia et options de Desplechin comme une actualisation de l'interrogation sceptique par les moyens du cinéma, conduisant ainsi à un mouvement d'interprétation infinie de textes énigmatiques: «Pour le cinéaste, les textes d'Emerson, de Nietzsche et de Cavell présentent une vérité paradoxale sur nos vies qui demande à être dépliée, déployée par le cinéma, mais quaucune explication ne peut atteindre si elle ne court le risque de produire à son tour une interprétation, un texte second ou troisième.» (id., p. 200).

8 «Pourquoi les films comptent-ils? Discussion entre Stanley Cavell et Arnaud Desplechin», op. cit., p. 2 I2. 
9 Voir Stanley Cavell, A la recherche du bonheur. Hollywood et la comédie du remariage, op. cit. It Happened One Night (Frank Capra, Etats-Unis, I934), The Awfull Truth (Leo McCarey, EtatsUnis, I937), Bringing Up Baby (Howard Hawks, Etats-Unis, I938), His Girl Friday (Howard Hawks, Etats-Unis, I940), The Philadelphia Story (George Cukor, Etats-Unis, I940), The Lady Eve (Preston Sturges, Etats-Unis, I94I) et Adam's Rib (George Cukor, Etats-Unis, I949) incarnent le «genre» de la comédie de remariage.

10 Voir Stanley Cavell, La Protestation des larmes. Le mélodrame de la femme inconnue, Paris, Capricci, 2012, trad. P. Soulat [première édition: Contesting Tears. The Hollywood Melodrama of the Unknown Woman, Chicago, The University of Chicago Press, I996]. Le «genre» du mélodrame de la femme inconnue repose sur le corpus suivant: Letter from an Unknown Woman (Max Ophüls, Etats-Unis, I948), Gaslight (George Cukor, Etats-Unis, I944), Now Voyager (Irving Rapper, Etats-Unis, I942), Stella Dallas (King Vidor, EtatsUnis, 1937). un scepticisme fondamental - ou, si l'on préfère, une «non-conformité», pour reprendre l'expression d'Emerson - qui informe également les analyses de films de Cavell. Mais ce qui nous paraît remarquable dans ce jeu d'homologie, c'est une approche comparable du récit filmique: l'herméneutique filmique de Cavell consiste à investir des comédies ${ }^{9} \mathrm{ou}$ des mélodrames populaires ${ }^{10}$ comme les grands textes philosophiques du $\mathrm{Xx}^{\mathrm{e}}$ siècle, tandis que les films de Desplechin articulent divers codes génériques à travers une multiplicité de références (filmiques, littéraires, philosophiques) qui surdéterminent ses films. Dans les deux cas, il nous paraît légitime de parler de surinterprétation - la relecture du cinéma hollywoodien ou les allusions à des films de genre se négociant et se rejouant sur le mode de la rhétorique du paradoxe. De manière générale, les films de Desplechin inscrivent ou déploient progressivement une série de signes symptomatiques qui ont une fonction d'embrayeur de la fiction, procédant par retournement de situations et bifurcation des lignes du récit (les relations triangulaires de couples et les conflits de pouvoir dans le milieu universitaire conduisant à l'hystérie et au délire paranoïaque dans Comment je me suis disputé..., tandis que dans La Sentinelle l'énigme politico-existentielle au sein de l'Europe de l'après-Guerre froide culmine dans des scènes d'assassinat dignes d'un film d'espionnage).

Dans La Sentinelle, la découverte d'une tête momifiée, glissée par l'espion Louis Bleicher dans la malle du protagoniste qu'il a interrogé et malmené à l'occasion d'un contrôle douanier, constitue le point de basculement du film: la tête fait effraction dans le récit, ou plutôt dans la valise de Mathias Barillet, cadrée en gros plan et enveloppée dans un voile telle une relique (le bruit du robinet qui coule se superposant à des sons grinçants de violon); lors de la même séquence, Barillet déballe la tête dans sa valise et, le plan suivant, se lève du lit, s'assied à même le sol, transi et atterré; le plan suivant, il découvre à nouveau d'un geste de la main la tête, posée sur le lit; coupe franche, on recadre Barillet qui répond au téléphone, avant de s'allonger, saignant du nez; avant-dernier plan de la séquence, la tête découverte est recadrée en plan rapproché sur une table, à côté d'un briquet; le plan final est cadré sur Barillet allongé sur le palier de la salle de bain, épanchant ses saignements. Corps du délit, la tête momifiée scelle le destin du protagoniste, les saignements de nez constituant un premier symptôme pathologique (la scène faisant par ailleurs allusion au Saint-Suaire). 


\section{Le personnage sceptique face à la "projection du monde "}

Le genre est mobilisé dans sa structure, mais il est évidé: de la comédie ou du mélodrame, ne restent plus que des bribes et des relations entre personnages, tant dans les lectures de films de Cavell - portant sur un mode de conversation qui culmine dans la création d'une nouvelle femme et la reconnaissance de l'altérité, interprétation par ailleurs vivement critiquée par Tania Modleski dans une perspective féministe ${ }^{11}$ que dans les films de Desplechin - mais dans ce cas, sous forme de promesses déçues. Desplechin actualise sur le plan filmique les lectures de Cavell: il constitue un nouveau «genre» de films, en retenant certains traits d'une catégorie de films qu'il isole et approfondit, la comédie de remariage ou le mélodrame de la femme inconnue ne constituant in fine que des fictions de genre.

Desplechin institue la rupture en norme, suivant la pente du scepticisme: niant toute certitude, le personnage cherche à faire l'expérience du monde extérieur, mais se heurte à l'existence fantomatique de l'image cinématographique. Attentif lecteur de Cavell - nous pensons à La Projection du monde ${ }^{12}$, souvent considéré comme le premier ouvrage qu'un philosophe a intégralement consacré au cinéma -, Desplechin part du postulat qu'au cinéma l'image est projetée, et que le «réel» est factice, impalpable ou intangible - tout au plus peut-il acquérir la densité de surface d'un miroir qui nous renvoie notre propre reflet, l'observateur étant luimême gagné par le doute. Dans La Projection du monde, le point où Cavell s'écarte irréductiblement de l'ontologie de l'image photographique synthétisée par Bazin - et qui lui permet contradictoirement de mobiliser sur le même niveau d'analyse l'approche iconographique de Panofsky ${ }^{13}$ porte sur l'existence physique paradoxale de l'image filmique dès lors qu'elle est projetée, et donc sur la relation de croyance que le spectateur entretient envers le film. Ceci s'applique analogiquement aux films de Desplechin, en bon interprète de Cavell: le personnage doit affirmer l'existence de sa propre image, face à un monde d'apparence qui est

11 Selon Tania Modleski, Cavell et par la même occasion les éditeurs de Critical Inquiry «perpétuent la condition même qu'ils analysent: ils participent à un système dans lequel les femmes ne sont pas reconnues, leur voix n'est pas entendue, et où leur identité demeure «inconnue `. Ainsi, la Femme peut être maintenue comme une énigme - le Continent noir que les hommes explorent, colo- nisent et «défendent), revendiquant des droits exclusifs sur un territoire qu'ils doivent absolument présenter comme vierge» (Tania Modleski, «Editorial Notes», Critical Inquiry, vol. $17, \mathrm{n}^{\circ} \mathrm{I}, \mathrm{au}-$ tomne I990, pp. 237-238). Cavell répond à ces critiques dans le même numéro (Stanley Cavell, «Editorial Notes», Critical Inquiry, vol. $17, \mathrm{n}^{\circ} \mathrm{I}$, automne 1990, pp. 238-244). Voir aussi Tania Modleski, Feminism without Women. Culture and Criticism in a «Postfeminist» Age, New York, Routledge, I99I, pp. IO-II.

12 Voir Stanley Cavell, La Projection du monde: réflexions sur l'ontologie du cinéma, Paris, Belin, I999, trad. Christian Fournier [The World Viewed: Reflections on the Ontology of Film, New York, Viking Press, 1971].

13 Voir Erwin Panofsky, «Style et matière du septième art» [1934], Trois essais sur le style, Paris, Le Promeneur, I997. trad. Bernard Turle, pp. Io9-I45. Il faut le souligner: Panofsky propose une analyse «iconographique» du cinéma, sans vraiment croire en la possibilité d'articulation d'une «iconologie» à travers le film (pour reprendre le distinguo réaffirmé par Thomas Y. Levin dans son introduction à la conférence de Panosfky qui, rappelons-le, a été prononcée à l'occasion de l'inauguration d'un Département film au MoMA; voir Thomas Y. Levin, «Un iconologue au cinéma. La théorie cinématographique de Panofksy», Les Cahiers du Musée national d'art moderne, $\mathrm{n}^{\circ}$ 59, printemps I997, pp. 35-74). L'ambition de Cavell, comme celle de Desplechin, est d'évoluer d'une iconographie filmique (mobilisant des figures stéréotypées) à une iconologie du cinéma (une figuration constituant un style singulier), pourrions-nous soutenir. 
14 Voir Virgil Vernier, "Christian Boltanski (I968-r999) et La Sentinelle d'Arnaud Desplechin. Les enfants du vingtième siècle face à la présence spectrale», dans Alain Brossat et Jean-Louis Déotte (éd.), L'Epoque de la disparition: politique et esthétique, Paris, L'Harmattan, 2000, pp. 315-342.

15 Voir James Joyce, Portrait de l'artiste en jeune homme, Paris, Gallimard, I992, trad. Jacques Aubert et Ludmilla Savitzky [The Portrait of the Artist as a Youg Man, New York, B. W. Huebsch, I9ı6].

16 Récusant la consistance du "genre» autofictionnel, tel qu'il a été revendiqué par Serge Doubrovski (Fils, Paris, Galilée, I977) et théorisé par Vincent Colonna (L'Autofiction. Essai sur la fictionnalisation de soi dans la littérature, thèse de doctorat, EHESS, I989), Genette considère que les autofictions «ne sont fictions que pour la douane: autrement dit, autobiographies honteuses » (Gérard Genette, Fiction et diction, Paris, Seuil, I99I, pp. 86-87, note 2). frappé d'une virtualité ontologique, celle de la projection entendue dans un sens à la fois cinématographique et psychanalytique. Qu'il s'agisse de Mathias Barillet - dont le patronyme renvoie au mécanisme du révolver et qui hérite d'une tête momifiée anonyme ${ }^{14}$ dont il cherche à attester l'identité et le passé - ou de Paul Dédalus - double du protagoniste joycien du Portrait de l'artiste en jeune homme ${ }^{15}$ qui nous invite à envisager le film comme une variation autour de la forme de l'autofiction, que nous pouvons caractériser à la suite de Genette comme une «autobiographie honteuse» ${ }^{16}$-, les personnages sont renvoyés à leur condition d'écran, à leur existence fantomatique ou spectrale. C'est là le sens du motif insistant, presque surréaliste, de la tête cadavérique dans La Sentinelle: signe indiciel (preuve) d'un disparu que les services secrets français s'attachent à supprimer une seconde fois, le crâne - malgré la référence à Hamlet, cité par l'acolyte de Barillet qui cherche à s'en emparer, et à une toile d'Holbein, sur laquelle nous reviendrons - témoigne d'une existence «projetée», limitée à sa seule visibilité - et qui constitue dès lors un fétiche, une tête réduite «comme les Jivaros» ainsi que le souligne Simon Acher (étudiant en médecine légale, joué par Fabrice Desplechin).

Précisons d'emblée que la pratique de l'autofiction est explicitement mise en scène dans les premières séquences de Comment je me suis disputé... comme une fausse autobiographie et une parole de cure qui conduit à l'invention de soi sur un mode mensonger, au gré d'une construction énonciative enchâssée: Paul Dédalus, maître-assistant en philosophie, revient sur une scène de son enfance, son alter ego de dix ans écrivant une autobiographie imaginaire («à la Stevenson» pour «tordre le cou à la vraie vie», précise-t-il), adressée contre son père; cette scène primitive est suivie d'une séquence centrée sur Paul Dédalus adulte, sur le divan du psychanalyste, qui explique la gêne qu'il a ressentie lorsque sa mère a découvert son journal et l'a sommé de s'expliquer... La métaironie constitue l'un des traits caractéristiques de cette autofiction qui tourne en dérision les rituels du monde académique et plus particulièrement d'un département d'épistémologie caustiquement mis en scène, le réductionnisme et le fonctionnalisme s'inversant ici en une économie morbide et un désir réprimé. Dans La Sentinelle, par contre, la fable est résolument ancrée dans l'Histoire, l'incipit du film renvoyant au partage de l'Europe convenu entre Staline et Churchill le 9 octobre 1944 - sur une feuille de papier hâtivement annotée et paraphée, dissimulée 
et remise à Churchill, que le motif du crâne, également divisé en deux hémisphères ou zones d'influence, vient prolonger. Desplechin entend ainsi dans son premier long métrage rompre avec le cinéma «intimiste» et «introspectif» français qui constitue son héritage immédiat ou qui lui est contemporain.

\section{La Vanité ou la structure de l'anamorphose}

L'énigme de La Sentinelle se cristallise autour de la tête momifiée d'un disparu politique, qui emblématise la guerre froide et la «frontière» après la chute du Mur de Berlin. L'un des chapitres du film (qui suit le découpage livresque suivant: Le Fantôme, Vivre avec, Le Remords, Les Ambassadeurs, Mon meilleur ennemi, Cavalier seul) souligne la référence explicite du crâne aux Ambassadeurs d'Holbein. Le renvoi au modèle de la Vanité fait sens en tant que dispositif de vision et accommodation du regard, plutôt que comme thématique ou clef d'analyse (en l'occurrence, la vanité des activités humaines, l'aveuglement face aux valeurs essentielles de ce monde, et l'insuffisance des arts et de la science). Sur ce point, nous souscrivons aux propos de Pierre Jaillou, tout en nous écartant de certains arguments de sa démonstration:

«La tête de mort ne se fait pas Vanité, mais renvoie à l'insignifiance du réel, d'autant que le cadavre n'est pas identifié [...]. [...] Evoquer les morts, ‘c'est, dans ce magma informe, apporter l'ordre et le nombre, distinguer des individus en les contraignant à se mettre en file, à la suite, à se présenter chacun à son tour et chacun pour soi, à parler en son nom et à se souvenir`. C’est le rôle du «messager〉 Mathias, qui s'extrait de la mélasse du réel pour s'en faire le médiateur. On ne sera plus collé à la toile, à la forme indéchiffrable qui gît aux pieds des Ambassadeurs d'Holbein - dont le titre est aussi celui d'un chapitre du film -, mais, prenant de la distance, on pourra lui donner forme: celle d'une tête de mort.» ${ }^{17}$

Néanmoins, cette tête de mort fait selon nous avant tout sens ou plutôt signe en tant que dispositif perceptif, comme le manifeste bien cette séquence qui a une fonction de «contre-champ» vis-à-vis de ce traumatisme initial: l'étudiante en histoire de l'art (Emmanuelle Devos) explique au médecin légiste le «schéma de la vision» dans la théorie de la perspective, ce qui conduit à une tentative ratée de séduction et de baiser volé de la part du jeune homme. Un faux raccord, la scène étant rejouée à travers un autre cadrage, souligne la dissymétrie des points de vue et
17 Pierre Jaillou, «Loin de moi», Eclipses, $\mathrm{n}^{\circ}$ 52, juin 2013 (Youri Deschamps, éd., «Arnaud Desplechin, l'intimité romanesque»), p. 45, p. 48. Jaillou cite Jean-Pierre Vernant, L'Individu, la mort, l'amour: Soi-même et l'autre en Grèce antique, Paris, Gallimard, 2005 [1989], p. 88 . 

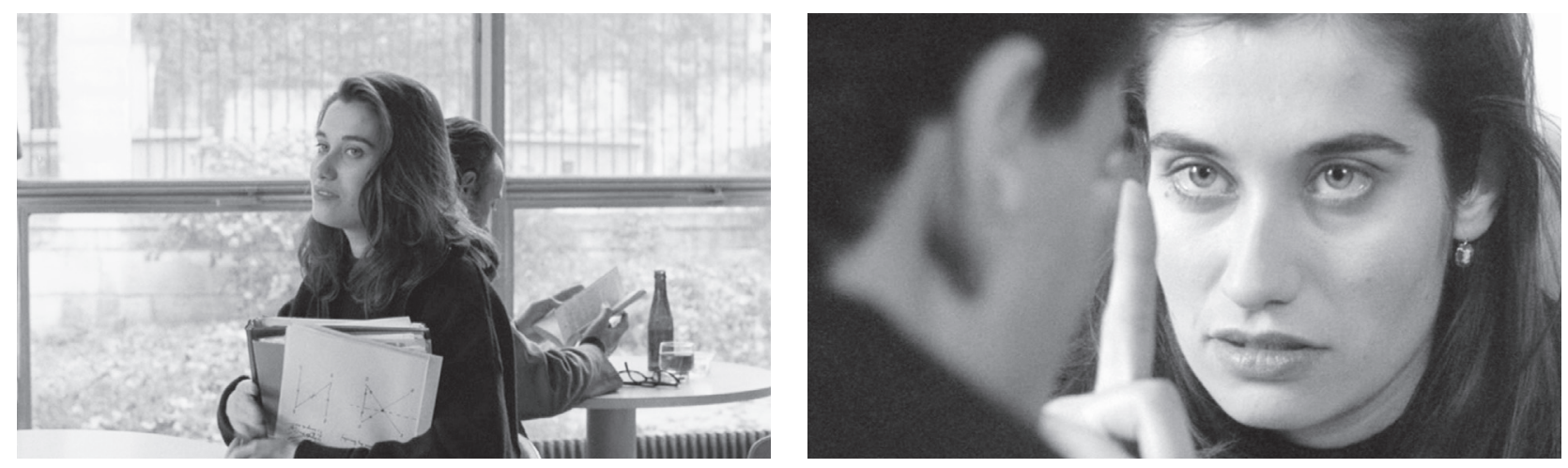

La Sentinelle, 1992

18 Voir le «Schéma L» de Lacan, notamment dans Jacques Lacan, «Le Séminaire sur «La Lettre volée»», Ecrits I, Paris, Seuil, ı966, p. 66. l'impossibilité de l'échange de regards (ou plutôt du baiser). Les schémas tracés sur la feuille de l'étudiante en histoire de l'art ne peuvent qu'évoquer le schéma de la relation imaginaire chez Lacan ${ }^{18}$ : l'entrée en scène du grand Autre (le lieu de la vérité) est bloquée, réprimée dans l'inconscient, le sujet se fixant sur cet autre (interprétée par Emmanuelle Devos) qui lui échappe pourtant. Le croisement des regards, ou la stéréoscopie de la vision, sont ici traduits en termes de relation à l'autre: à savoir le graphe du désir, qui est vécu sur le mode de la défection et de la désillusion.

Parmi les différents motifs inscrits sur la toile d'Holbein, Desplechin choisit d'autonomiser le globe terrestre, inscrivant la question des frontières (tout en prolongeant la forme du crâne et les références filées à Shakespeare), et bien évidemment la tête de mort qui n’est identifiable

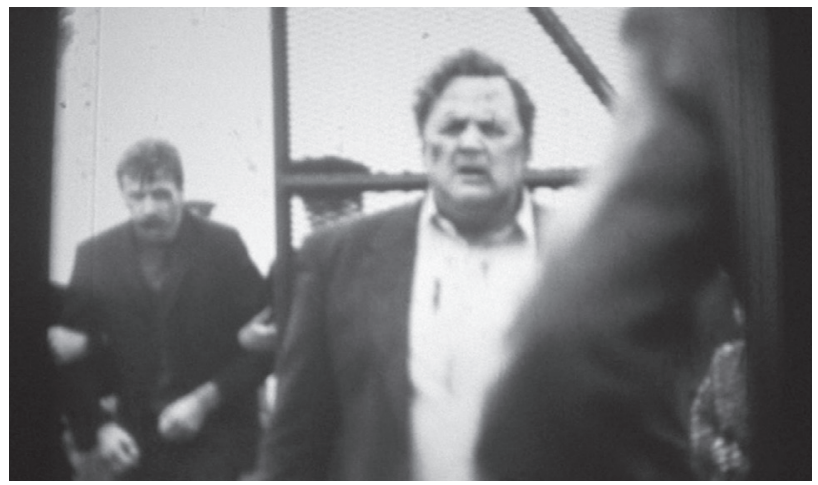


qu'à la condition que le spectateur du tableau modifie son point de vue. Desplechin nous invite donc à accommoder différemment notre regard, la tête de mort ne représentant pas tant une béance dans la représentation (pour poursuivre cette topique lacanienne) qu'une voie d'accès à une vision anamorphosée, oblique, secrète: une autre scène qui est évoquée à travers la projection d'un film Super-8 sur lequel apparaît l'espion (ou le passeur) qui réintroduit furtivement le crâne dans l'Europe d'après la Chute du Mur. L'enjeu du film est rejeté hors-champ: il est inutile de disséquer et authentifier le crâne (ou alors on ne sort pas de l'ontologie bazinienne de l'image photographique comme embaumement, voire de la réduction de l'image à une preuve), la représentation ne se déchiffrant non pas tant dans une relation imaginaire à l'autre (réinvestie dans le champ du symbolique, ici le schéma de la vision ou les codes génétiques du prisonnier politique) qu'à travers un accès trouble à la relique d'un événement qui a fait Histoire, et qui insiste, à l'image du réel - autrement dit, si l'on suit Lacan: ce contre quoi on se heurte (d'où les saignements de nez récurrents de Mathias Barillet). Pour le dire autrement, la tête cadavérique obnubile l'attention du protagoniste du film, qui ne parvient plus à faire le point sur les événements qui l'environnent - grande bourgeoisie parisienne, milieux retors de la diplomatie, mais aussi les étudiants en médecine et son entourage le plus proche, dont sa sœur-, l'anamorphose gagnant le monde de la représentation tout en laissant à découvert son point obscur (le crâne), dont la présence insoutenable, presque obscène dans son objectité, devient aveuglante.

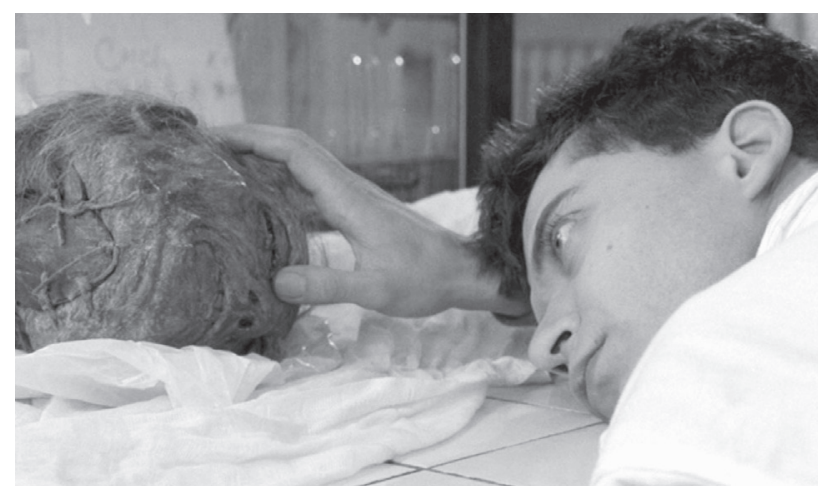


19 «Laurence Briaud, la monteuse du cinéaste sur plusieurs de ses films, explique que la première étape du montage consiste chez Desplechin à « réaliser une sorte de best-of, c'est-à-dire monter à la file [...] les meilleures prises d'une même séquence), et que le cinéaste n'hésite pas à conserver dans le montage final plusieurs versions d'une même scène» (Céline Saturnino, «Etats d'âmes, état des corps», Eclipse, op. cit., p. 24).

20 Voir Saad Chakali, «Les héritiers symptomatiques», Eclipses, op. cit., pp. I22-I30.

21 George Didi-Huberman, Devant le temps. Histoire de l'art et anachronisme des images, Paris, Minuit, 2000, p. 22I, cité par Saad Chakali, op. cit., p. I24 (c'est nous qui tronquons la citation).

22 Freud décline la proposition de Pabst, soumise par le biais d'Abraham: «Le projet ne me plaît guère. Je ne tiens pas pour possible de présenter nos abstractions de façon plastique.» (Lettre de Sigmund Freud à Karl Abraham, I925, cité par Patrick Lacoste, L'Etrange cas du Professeur M., Psychanalyse à l'écran, Paris, Gallimard, I990, p. 34).

23 Voir Hanns Sachs, Psychoanalyse. Rätsel des Unbewussten, Berlin, Lichtbild-Bühne, I926, traduit par $\mathrm{Pa}$ trick Lacoste, L'Etrange cas du Professeur M., op. cit., pp. 27I-294.

24 Voir Hanns Sachs, «Psychologie du film», Positif, n 74, mars i966, pp. 67-73 [première publication: «Film Psychology», Close Up, $\mathrm{n}^{\circ} \mathrm{I7}$, novembre 1928 , pp. 8-I5].

\section{Symptomatologie de la vie quotidienne}

Chez Desplechin, les allusions filmiques, littéraires et philosophiques participent à une logique de construction du récit par série de renvois et d'échos qui redoublent la structure apparente du film, l'écriture scénaristique répondant à une «règle du jeu» qui est proche par certains aspects de la littérature à contrainte (ce serait cependant forcer le trait que de convoquer ici l'exercice de style oulipien), tout en s'ouvrant à l'indétermination et à l'improvisation (variantes du scénario, mais aussi variations des mêmes plans lors du tournage, conservées au montage $\left.{ }^{19}\right)$. Précisons toutefois que les films de Desplechin ne procèdent pas seulement par masques ou par références, mais aussi par la constitution d'imagessymptômes, pour reprendre la terminologie de Georges Didi-Huberman que Saad Chakali applique à l'univers filmique de Desplechin ${ }^{20}$ :

«[...] qu'est-ce, en effet, qu'un symptôme, sinon le signe inattendu, non familier, souvent intense et toujours disruptif, qui annonce visuellement quelque chose qui n'est pas encore visible, quelque chose que nous ne connaissons pas encore? Si l'image est un symptôme - au sens critique et non clinique du terme -, si l'image est un malaise dans la représentation, c'est qu'elle indique un futur de la représentation [...].» ${ }^{21}$

Les films de Desplechin répondent à une logique qui est de l'ordre de la symptomatologie, sans qu'elle n'appelle pour autant un «futur de la représentation ». Sans craindre le forçage interprétatif, nous proposons d'envisager Comment je me suis disputé... comme une version «postmoderne» de Geheimnisse einer Seele (Les Mystères d'une âme, Allemagne, I926) de Georg W. Pabst, dont le scénario repose sur les conseils de Karl Abraham, puis de Hanns Sachs après son décès. Rappelons que, contre l'avis de Freud qui s'oppose à la «figuration» de l'inconscient ${ }^{22}$, Pabst retrace à l'écran, grâce aux apports d'Abraham et de Sachs - parmi les premiers disciples restés fidèles au «père de la psychanalyse»-, le processus de la cure analytique, sur un plan par ailleurs tout à fait littéral et illustratif, dans une visée didactique. Sachs ne participe pas seulement au scénario du film de Pabst, il rédige également une brochure explicative ${ }^{23}$ qui «interprète» Les Mystères d'une âme à travers une grille de lecture symptomatologique - qu'il explicite et théorise par la suite ${ }^{24}$. En effet, si les actes manqués et les mots d'esprit structurent le scénario des Mystères d'une âme, ils sont également inscrits selon Sachs dans des scènes apparemment anodines de films, en l'occurrence le mouvement 
de détournement d'un garde avant l'ordre de la fusillade sur Le Cuirassé Potemkine (Sergueï Mikhaïlovitch Eisenstein, URSS, I925), le geste d'un gardien qui repousse un cafard au fond du bol de soupe d'un prisonnier dans La Mère (Vsevolod Poudovkine, URSS, I926), et le geste amoureux d'une femme qui manipule la cravate de son amant qui est sur le point de la délaisser dans Three Women (Trois Femmes, Ernst Lubitsch, Etats-Unis, I924). Cette logique symptomatique, Sachs l'analyse en termes de lapsus ou de manifestation apparente d'un fantasme latent: l'acteur exprime des «événements psychiques qui sont en deçà ou au-delà du langage» par le biais de «réflexes» et de «légères impropriétés du comportement, décrites par Freud comme des actes symptomatiques». Autrement dit, des «mouvements en eux-mêmes insignifiants et triviaux», tels que la perte d'un objet, un jeu inconscient avec un objet ou encore l'oubli d'une action, expriment le travail de l'inconscient à travers un moyen d'expression cinématographique. Ces lapsus déterminent la direction du jeu et l'écriture scénaristique dans les films de Desplechin, qui sont centrés sur un processus de dénégation (un refus de participer à l'ordre dominant, un retrait hors du monde de la diplomatie, une propension aux ruptures amoureuses, un déni des règles académiques). La scène de Comment je me suis disputé... où Paul Dédalus (Mathieu Amalric, dont la performance porte véritablement le film) écrase entre les montants d'une porte la main de son collègue Rabier (Michel Vuillermoz), distant et méprisant, qui refuse de le (re)connaître, peut être envisagée comme une mise en abyme ou un commentaire qui porte sur ce jeu «symptomatologique». L'«acte

25 Ce dicton s'origine dans les Entretiens de Confucius: "Ce qui est contraire au rituel, ne le regarde pas, ne l'écoute pas; ce qui est contraire au rituel, n'en parle pas et, à plus forte raison, n'y commets pas tes actions» (Anne Cheng, Entretiens de Confucius, livre XII, Paris, Seuil, i98I, p. 95).

26 Stanley Cavell, «En quoi consiste le scandale du scepticisme?», Philosophie: le jour d'après demain, Paris, Fayard, 20II, trad. N. Ferron, p. I67 [Philosophy the Day after Tomorrow, Cambridge, Mass./ Londres, Harvard University Press, 2005]. Notons qu'Elise Domenach (op. cit.) renvoie à ce passage de l'ouvrage de Cavell dans son analyse d'Un conte de Noël.

27 «Le titre, programmatique, se présente dans son incomplétude comme une phrase à construction boiteuse: il manque quelque chose [...], quelque chose de l'ordre de l'innommable, de l'indicible. [...] Soit le verbe « disputer > prend une valeur intransitive, soit son complément est une variable, comme le laisse envisager l'idée d'interchangeabilité des personnages dans la structure du scénario. La parenthèse 〈(ma vie sexuelle)〉 peut tout autant être lue comme la promesse du dénouement que comme un simple élément de résolution. Le titre prévu à l'origine était plus simple: Comment je me suis disputé avec Rabier. [...] Le nom de Rabier fait résonner un ami de promotion de Desplechin, Eric Barbier; il évoque plus directement un dessinateur vendéen, précurseur de la bande dessinée, qui travaillait la représentation des animaux, ce qui expliquerait la présence du singe auprès de Rabier, qui le tient à la fois par une chaîne et par la main.» (Yola Le Caïnec, «La vie comme un roman», Eclipses, op. cit., p. 54, p. 56). manqué» permet de laver l'affront, de réparer l'injustice tout en faisant écho au long travail d'analyse explicité par Pabst et Sachs: chez Desplechin, sur un mode ouvertement comique et outrancier, l'instantanéité de l'acte supplée la cure analytique, qui n’aboutit pas, le professeur d'épistémologie ayant refusé de saluer à deux reprises son collègue (la première fois, par crainte de souillure, celui-ci ayant manipulé le cadavre de son singe coincé sous un radiateur, la seconde fois pour afficher son dédain, feignant de ne pas le reconnaître). Ajoutons seulement que le jeu de la citation ou de l'allusion vient encore surdéterminer la scène, le singe de compagnie de Rabier renvoyant à la parabole des trois singes, muets, aveugles et sourds - ou, pour renvoyer à la sagesse populaire, d'après Confucius: «Devant l'impolitesse, ne pas regarder, ne pas écouter, ne pas parler, ne pas bouger» ${ }^{25}$. Du point de vue de Paul Dédalus, qui cherche à accorder son pardon à Rabier alors que celui-ci ne le sollicite aucunement, l'enjeu est de renouer le fil de sa vie avec autrui, conformément à certains propos de Cavell:
«[...] dans la négation ordinaire sous ses diverses formes qui surgit au fil de ma vie avec autrui - dans un moment d'irritation, une rancune persis- tante, un accès soudain de ressentiment, un regard dur, un témoignage malhonnête, un mensonge que l'on dit, un mensonge que l'on croit, un silence gêné, la peur de l'engloutissement, le fantasme de la solitude ou de l'autodestruction, - le problème est de reconnaître que c'est moi qui suis en train de nier l'autre, c'est de comprendre que je porte le chaos en moi. C'est là que réside le scandale du scepticisme à l'égard de l'existence des autres; je suis le scandale.» ${ }^{26}$

Quoi qu'il en soit, Amalric est un acteur révélé par Desplechin, en ce sens que son corps est traversé par une succession de signes symptomatiques qui le trahissent: il appelle au déchiffrement analytique, les symptômes révélant - ou créant - le fantasme, celui d'être aimable et aimé. En ce sens, Desplechin nous propose une «comédie de la rupture et du remariage», le protagoniste visant à l'accomplissement de la séparation - ce théâtre de la dispute donnant naissance à un nouvel homme, selon une dynamique résolument solipsiste. Or, on n'a pas manqué de le relever, la construction paradoxale du titre du film traduit son instabilité énonciative, oscillant entre une forme transitive et une tournure intransitive ${ }^{27}$, portant le trouble sur la distinction entre les places (grammaticales) du sujet et de l'objet. Frédéric Rabier, de toute évidence, désigne 
un camarade de promotion de Desplechin à la Fémis, Eric Barbier. Mais l'objet visé (ou le sujet désigné) est également l'alter ego du protagoniste (du cinéaste?), Paul Dédalus, dont le but est la création de soi à travers l'acceptation de l'autre, par le biais de l'épreuve de la rupture et du remariage - car, il faut le rappeler, les trois singes de la sagesse chinoise «représentent ce qu'il faut respecter pour garantir l'harmonie», c'est-àdire «la paix des ménages» selon «le sens commun en Occident» ${ }^{28}$. Là encore, nous retrouvons la trame des comédies de remariage, même si le protagoniste incarné par Amalric ne dépasse pas le stade de la rupture (avec son ancienne compagne, avec ses ressentiments à l'égard de Rabier), exemplairement lors de sa chute dans l'escalier de la faculté - acte manqué s'il en est - qui déclenche sa volonté de réconciliation unilatérale (comme si elle s'était fixée sur un mauvais objet, Rabier plutôt qu'Esther, qui a littéralement perdu la parole, devenue aphone). Ajoutons que les divisions internes du sujet empêchent la formation d'un moi qui se distinguerait de l'autre (ce que l'on appelle communément: le stade du miroir), et que la disputatio ne parvient pas à s'articuler en conversation avec autrui: le dialogisme se réduit ici à un monologue entre le sujet et l'image qu'il tend à projeter à son entourage et à lui-même.

La tentative de rupture entre Paul (Mathieu Amalric) et Esther (Emmanuelle Devos) constitue une séquence nodale dans Comment je me suis disputé..., qui scelle la difficulté de Paul à accéder à un mode dialogique de conversation permettant d'instituer la relation de couple à partir de la reconnaissance d'autrui. La première partie de la séquence se déroule dans un café, avec le récit de la rupture rapporté en voix over, suivant une stratégie discursive à laquelle François Truffaut a régulièrement recours avec Jean-Pierre Léaud. Le décalage entre la bande-son et la bande-image (gagnée par un silence qui se substitue au son in dans le café) permet d'énoncer la rupture sur un ton impersonnel, à travers un discours à la troisième personne - isolation soulignée par un champ/ contrechamp de profil qui concourt à séparer le couple qui était auparavant réuni dans le même cadre. La séquence se poursuit dans la chambre de Paul, Esther venant voir Paul afin de comprendre et d'infléchir sa décision. L'explication tourne au conflit, Paul mobilisant la parole en recourant à divers effets de discours. Le litige, qui se noue autour du fait de «porter la responsabilité de l'autre», dévie sur le narcissisme de Paul (sa dépression, sa crainte d'être un raté) avant d'aboutir sur sa liaison avec
28 René Barbier, La Pensée chinoise en Occident. Métissage des cultures et ouverture de la conscience, 2013, publication en ligne, p. 25. Spécialiste de la «rechercheaction existentielle», René Barbier est l'auteur notamment de La Rechercheaction dans l'institution éducative, Paris, Gauthier-Villars, I977; «La Rechercheaction existentielle», Pour, $\mathrm{n}^{\circ}$ 90, juinjuillet 1983, pp. 27-31; La Rechercheaction, Paris, Anthropos, I996. 

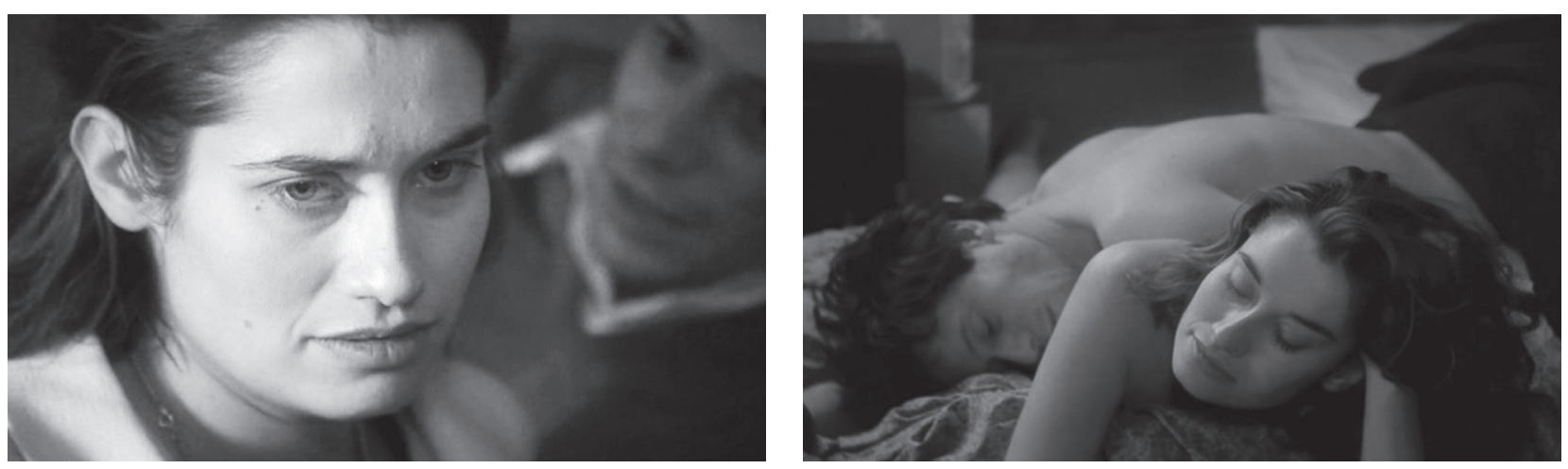

Comment je me suis disputé...

(ma vie sexuelle), 1996

une autre femme. Les plans, ne respectant pas les raccords de continuité, s'enchaînent en suivant différents rythmes, ponctués par de brèves ellipses, qui permettent d'alterner librement entre les divers états affectifs du couple. La nuit tombée, la troisième partie de la séquence s'ouvre sur un plan d'Esther dans la cuisine, qui fume face à un cendrier plein. La conversation se focalise sur la possibilité de partager un appartement; mais rapidement, le ton s'envenime, tandis que l'énonciation évolue de l'échange direct de paroles à un mode rapporté de discours. En effet, Esther demande à Paul quelles paroles elle devrait prononcer pour qu'il l'aime encore; elle répète alors ses propos, le confortant dans ce qu'il croit vouloir entendre. Le dialogue qui s'échange dans la chambre constitue une variation sur la comédie de remariage, tout en invalidant ses conditions de possibilité d'existence - les personnages recourant au discours indirect, c'est-à-dire n'assumant pas la position grammaticale du je. Cette dépersonnalisation de l'énonciation culmine à travers la superposition de bribes de discours, en voix in et over, qui reprennent et brouillent les paroles de Paul, parachevant d'aliéner le discours, la focalisation interne de la scène, du point de vue d'Esther, traduisant son étourdissement et son abasourdissement. Quand le dialogue reprend sur un mode plus naturaliste, Esther s'écarte de Paul, le mettant à distance, avant de parler à la première personne, pour lui déclarer son amour. Paul, par contre, continue à s'exprimer par le truchement de paroles qui ne lui sont pas propres, jusqu'à soutenir qu'il ne l'aime pas - avant de se reprendre, déclarant sa flamme et amorçant une litanie de poncifs, qu'Esther interrompt violemment. La séquence ayant atteint son paroxysme - la crise 
ou la rupture étant désormais énoncées et vécues par Esther -, le couple peut momentanément se réconcilier; après une nouvelle ellipse, la séquence s'achève sur les amants qui se disputent au lit.

Ici, Desplechin transpose, actualise et en fin de compte inverse le mode de conversation qui prévaut dans les comédies de remariage hollywoodiennes, en mettant en scène l'ambivalence conflictuelle de Paul qui, dans son déni de l'autre, se constitue en sujet destitué et dépourvu de tout désir. L'exercice de style n’est pas seulement virtuose, il éclaire encore d'une lumière crue, presque clinique, le clivage du sujet et le mécanisme de dénégation d'autrui au sein d’un milieu spécialisé dans la production d'un savoir qui est donné à voir comme une vanité.

La dynamique intertextuelle que Desplechin met en jeu dans l'ensemble de ses films, qu'elle se négocie à travers le registre du comique dans Comment je suis disputé... ou celui d'une «inquiétante étrangeté» qui déstabilise l'horizon d'attente du film d'espionnage dans La Sentinelle, participe à une logique d'appropriation, et non à celle du «pastiche» ${ }^{29}$ : le genre est retravaillé et déstabilisé dans ses soubassements logiques, et non pas mis à distance ou convoqué sur un mode ostensiblement citationnel. Et si la parodie - que Linda Hutcheon ${ }^{30}$ propose de substituer au mode du pastiche, en prenant à revers les thèses de Frederic Jameson qui portent sur une forme dépolitisée de convocation d'un «style» - est omniprésente dans Comment je me suis disputé..., elle est réactivée sur un mode sceptique, n'étant jamais assurée de son efficience politique qui repose sur le rire et la subversion. La stratégie de l'appropriation, il faut le souligner, constitue un authentique travail de sape qui peut en venir à nier sa propre forme dérivative, à l'image des films de Desplechin où le sujet «se dispute» jusqu'à se destituer de sa propre identité (voir à cet égard l'image-symptôme de Paul Dédalus / Mathieu Amalric hébété, qui a momentanément perdu l'usage de la parole et de la raison, se tenant au milieu d'un chemin - nel mezzo del camin ${ }^{31}$, etc. -, après s'être débarrassé du cadavre, coincé sous un radiateur, du singe de Rabier).
29 Sur la forme «postmoderniste» du pastiche, voir Frederic Jameson, « Postmodernism, or the Cultural Logic of Late Capitalism», New Left Review, $\mathrm{n}^{\circ}$ I46, juillet-août I984, pp. 59-92.

30 Voir Linda Hutcheon, A Theory of Parody: The Teachings of TwentiethCentury Art Forms, Urbana, University of Illinois Press, 2000.

31 Voir le début du «Chant I» de L'Enfer: «Au milieu du chemin de notre vie, ayant quitté le chemin droit, je me trouvai dans une forêt obscure»... 